\title{
Small, dark, and heavy: But is it a black hole?
}

\author{
Matt Visser*t \\ School of Mathematics, Statistics, and Operations Research, Victoria University of Wellington, \\ New Zealand \\ E-mail: matt.visser@msor.vuw.ac.nz
}

\section{Carlos Barceló}

Instituto de Astrofísica de Andalucía, CSIC, Camino Bajo de Huétor 50, 18008 Granada, Spain

E-mail: carloseiaa.es

\section{Stefano Liberati}

International School for Advanced Studies (SISSA), Via Beirut 2-4, 34014 Trieste, Italy

and INFN, Sezione di Trieste

E-mail: liberatiesissa.it

\section{Sebastiano Sonego}

Università di Udine, Via delle Scienze 208, 33100 Udine, Italy

E-mail: sebastiano.sonegoeuniud.it

Astronomers have certainly observed things that are small, dark, and heavy. But are these objects really black holes in the sense of general relativity? The consensus opinion is simply "yes", and there is very little "wriggle room". We discuss one of the specific alternatives.

Black Holes in General Relativity and String Theory

24-30 August, 2008

Veli Losinj, Croatia

\footnotetext{
* Speaker.

${ }^{\dagger}$ This research was supported by the Marsden Fund administered by the Royal Society of New Zealand
} 


\section{Introduction}

Do alternatives to standard classical black holes exist? Can one "mimic" a black hole with arbitrary accuracy? There is a rather limited set of (arguably) viable alternatives:

- Quark stars [1], Q-balls [2], strange stars [3].

- Boson-stars [4].

- Gravastars: Mazur-Mottola variant [5], and Laughlin et al. variant [6].

- Fuzz-balls: Mathur et al. variant [7], and Amati variant [8]. (See [9] for a survey.)

- Dark stars/quasi-black holes [10, 11]. (For related ideas, see [12, 13]).

While there are close inter-relationships between these various models, in this article we will specifically focus on our own proposal $[10,11]$, and give an informal overview of the situation. We shall re-assess and (hopefully) re-invigorate an old line of argument: What effect does quantum physics have on the collapse of a classical star? Is semi-classical collapse [10, 12, 13, 14, 15] qualitatively different from classical collapse [16]?

In general we can certainly write

$$
G_{a b}=8 \pi\left\langle\psi\left|\hat{T}_{a b}\right| \psi\right\rangle,
$$

and separate the expectation value of the stress-energy-momentum tensor into a contribution from some suitably chosen vacuum state, plus a contribution from the excitations above that vacuum state. For instance, for an uncollapsed star

$$
G_{a b}=8 \pi\left(T_{a b}^{\text {classical }}+\left\langle 0\left|\hat{T}_{a b}\right| 0\right\rangle\right) .
$$

The vacuum polarization effect $\left\langle 0\left|\hat{T}_{a b}\right| 0\right\rangle$ is utterly negligible in an ordinary uncollapsed star. (This, after all, is why we can get away with just solving the classical Einstein equations most of the time.) Does this remain true during collapse? Even if the vacuum polarization does remain small, it might still have a significant effect on the location and/or existence of event horizons $[14,15]$.

Now this point of view, while certainly historically respectable, does deviate significantly from the present "consensus opinion", at least in the general relativity community, so before one gets started there are a number of preliminary issues that should be dealt with.

\section{The Fulling-Sweeny-Wald (no-singularity) theorem}

There is a widespread feeling in the general relativity community that semiclassical quantum back-reaction effects are always small, and never enough to significantly alter the classical picture of collapse to a black hole. (See figure 1 for an appropriate Carter-Penrose diagram.) When pushed, members of this community ultimately point to the Fulling-Sweeny-Wald no-singularity theorem [17] as the basis for this assertion.

Phrasing the Fulling-Sweeny-Wald theorem rather loosely: "In quantum field theory on a curved spacetime everything is finite at an event horizon, and all the way down to either a singularity or a Cauchy horizon." The technical content of this theorem is based on showing that the 


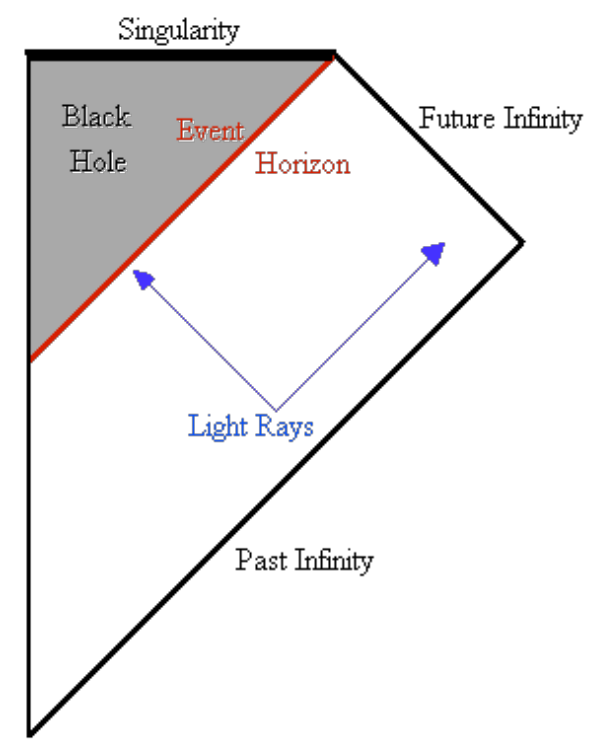

Figure 1: Standard Carter-Penrose diagram for an astrophysical black hole formed via stellar collapse.

Hadamard form of the two-point function for a quantum field is not affected by the presence of an event horizon. (So for a Hadamard quantum state, the renormalized stress-energy tensor [RSET] is automatically finite.) Unfortunately, for the question we want to raise, the Fulling-Sweeny-Wald theorem also "begs the question", for what it shows is that if an event horizon forms, then the quantum field theory is well behaved there. But this is not the same as showing that an event horizon will naturally form in semiclassical collapse: A finite but very large contribution from the vacuum polarization term $\left\langle 0\left|\hat{T}_{a b}\right| 0\right\rangle$ in equation (1.2), while perfectly in agreement with the theorem, would significantly alter the dynamics of collapse. In fact, compact horizonless objects, and/or naked singularities, are also fully compatible with both the hypotheses and the conclusions of the Fulling-Sweeny-Wald theorem.

\section{Horizons and the choice of the quantum vacuum state}

Our specific proposal for a black hole mimic is ultimately related to the appropriate choice for the quantum vacuum state $|0\rangle$. Common candidates states are:

Boulware vacuum: This is singular at any Killing horizon. The renormalized stress-energymomentum diverges as $r \rightarrow 2 m$.

Unruh vacuum: This is designed to be well-behaved at any future Killing horizon, so in particular the renormalized stress-energy-momentum is finite there.

Neither of these quantum states contain particles in the vicinity of past null infinity $\mathscr{I}^{-}$. Accordingly, the key constraint that we shall adopt for our global vacuum state is that it contain no particles in the vicinity of $\mathscr{I}^{-}$. Additionally, far in the past, near past timelike infinity $i^{-}$, when spacetime is 
static (and nearly Minkowskian), the quantum state should exhibit properties qualitatively similar to those of the Boulware vacuum. This demand is physically appropriate, since the Boulware vacuum is the one that best describes physics in the presence of a static self-gravitating object. Note that if we were instead to choose a state that in the asymptotic future behaves like the Unruh vacuum, then this would presuppose the formation of a horizon, which however is precisely the issue we wish to investigate. Such a choice would anyway amount to making a teleological statement. Apart from these particular issues, there is an increasing consensus, or at the very least a suspicion, within the general relativity community that event horizons are simply the wrong thing to be looking at. Sometimes apparent horizons [18, 19, 20] (or better yet, dynamical horizons [21], or trapping horizons [22]) are better candidates for characterizing the black hole. (See also [23, 24, 25].)

\section{Our specific calculation}

The metric for the spacetime of a spherically symmetric collapsing body can be written in Schwarzschild coordinates as

$$
g=-e^{-2 \Phi(r, t)}(1-2 m(r, t) / r) \mathrm{d} t^{2}+\frac{\mathrm{d} r^{2}}{1-2 m(r, t) / r}+r^{2}\left(\mathrm{~d} \theta^{2}+\sin ^{2} \theta \mathrm{d} \varphi^{2}\right) .
$$

In these coordinates an apparent/dynamical/trapping horizon, if it forms, is characterized by

$$
\frac{2 m(r, t)}{r}=1
$$

In contrast, an event horizon, if it forms, can only be found by back-tracking from future null infinity, $\mathscr{I}^{+}$.

In the standard classical conformal Carter-Penrose diagram for the collapse process, figure 2, one truncates the diagram at the centre, $r=0$, and modes of any field residing on the spacetime are said to "bounce" off the centre. A central part of the analysis is then to relate the affine null parameter $W$ on $\mathscr{I}^{-}$to the affine null parameter $u$ on $\mathscr{I}^{+}$via some function $W=p(u)$, which thus encodes a good fraction of all the physics.

For technical reasons we prefer to work with a more "symmetric" version of the CarterPenrose causal diagram. In this version, figure 3 , modes propagate straight through the centre of the collapsing star (located at $r=0$ ), so that $\mathscr{I}_{\text {left }}^{-}$is connected to $\mathscr{I}_{\text {right }}^{+}$, and vice versa. Affine null coordinates on $\mathscr{I}^{-}$are now related to affine null coordinates on $\mathscr{I}^{+}$by:

$$
U=p(u) ; \quad W=p(w) .
$$

Furthermore, we shall work in a $1+1$ dimensional truncation of $3+1$ dimensional spacetime in order to keep the calculation tractable. It will then be convenient to extend the coordinate $r$ in such a way that on the right-hand part of the diagram $r>0$, while in the left-hand part $r<0$.

For a massless real scalar field, one can expand the field operator using a set of modes that near $\mathscr{I}_{\text {left }}^{-}$take the form

$$
\varphi_{\Omega}(r, t) \approx \frac{1}{(2 \pi)^{3 / 2}(2 \Omega)^{1 / 2}|r|} \mathrm{e}^{-\mathrm{i} \Omega U}
$$




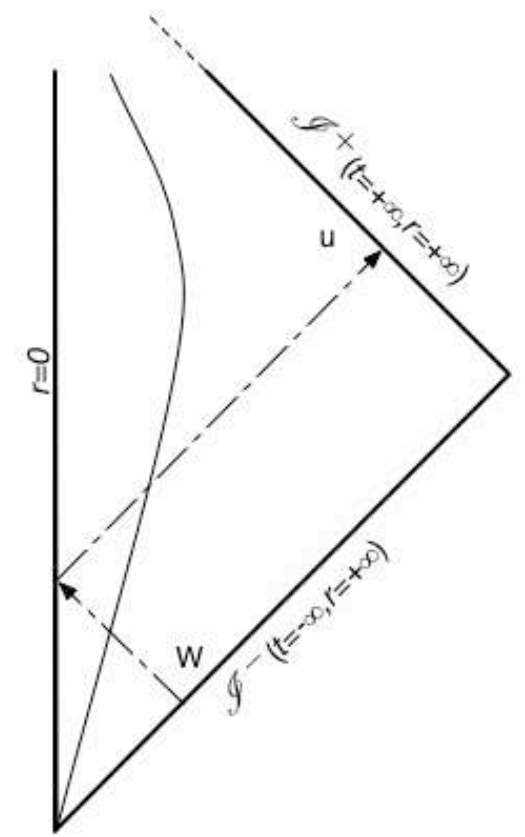

Figure 2: Standard Carter-Penrose diagram for the collapse process: Modes "bounce" off the centre of the star. We deliberately leave the "late time" portion of the diagram vague and ambiguous - since we do not want to pre-judge what the system settles down to.

with $\Omega>0$; these are appropriate to define particles in the asymptotic past, before collapse takes place. Neglecting backscattering, such modes take, near $\mathscr{I}_{\text {right }}^{+}$, the form

$$
\varphi_{\Omega}(r, t) \approx \frac{1}{(2 \pi)^{3 / 2}(2 \Omega)^{1 / 2} r} \mathrm{e}^{-\mathrm{i} \Omega p(u)} .
$$

However, near $\mathscr{I}_{\text {right }}^{+}$, the modes appropriate for defining particles are not the $\varphi_{\Omega}$, but others that we denote $\psi_{\omega}$, with the asymptotic form

$$
\psi_{\omega}(r, t) \approx \frac{1}{(2 \pi)^{3 / 2}(2 \omega)^{1 / 2} r} \mathrm{e}^{-\mathrm{i} \omega u}
$$

Hence, the state which does not contain particles on $\mathscr{I}^{-}$(defined using the modes $\varphi_{\Omega}$ ) turns out to contain particles on $\mathscr{I}^{+}$(defined using the $\psi_{\omega}$ ), provided that $p(u)$ is a non-trivial function, such that a $\varphi_{\Omega}$ mode contains negative-frequency contributions when Fourier-analysed in terms of the $\psi_{\omega}$. Defining the $u$-dependent frequency on $\mathscr{I}^{+}$,

$$
\omega(u, \Omega)=\dot{p}(u) \Omega,
$$

associated with a $\varphi_{\Omega}$ mode, one can see that mode excitation takes place provided that the adiabatic condition,

$$
\frac{|\dot{\omega}(u, \Omega)|}{\omega^{2}} \ll 1,
$$




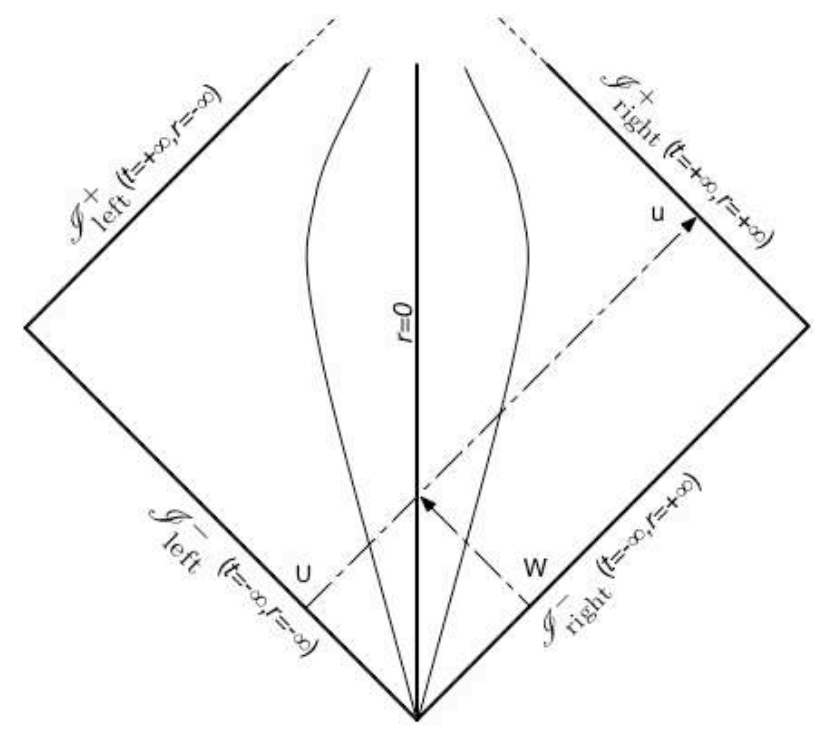

Figure 3: Our preferred Carter-Penrose diagram for the collapse process: Modes propagate straight through the centre of the star. We deliberately leave the "late time" portion of the diagram vague and ambiguous since we do not want to pre-judge what the system settles down to. $U-W$ coordinates are best in the bottom corner of the diagram, in fact near all of $\mathscr{I}^{-}$. In contrast, $u$-W coordinates are best in the upper-right region, near $\mathscr{I}_{\text {right }}^{+}$. (And $U-w$ coordinates are best in the upper-left region, near $\mathscr{I}_{\text {left }}^{+}$.)

is violated. This occurs for frequencies smaller than

$$
\Omega_{0}(u) \sim \frac{|\ddot{p}(u)|}{\dot{p}(u)^{2}},
$$

which can then be thought of as a frequency marking, at each instant of retarded time $u$, the separation between the modes that have been excited (those with $\Omega \ll \Omega_{0}$ ) and those that are still unexcited $\left(\Omega \gg \Omega_{0}\right)$. Intuitively, one may think that there is still an infinite "reservoir" of highenergy Boulware-like modes, and that if mode excitation is not sufficiently rapid they will make a potential obstruction to horizon formation. Indeed, calculations in static models [26] show that such modes lead to an energy condition-violating renormalised stress-energy-momentum tensor that diverges as one approaches the horizon. We feel however, that this is far too naïve a picture, being based on results obtained in static spacetimes, and that it can be taken at best as a hint that one should carefully check how the renormalised stress-energy-momentum tensor behaves when the horizon is just about to form. So, let us now turn to a calculation that takes dynamics explicitly into account.

The spacetime metric can be written using either the set of coordinates $(U, W)$, or $(u, W)$ :

$$
g=-C(U, W) \mathrm{d} U \mathrm{~d} W=-\tilde{C}(u, W) \mathrm{d} u \mathrm{~d} W
$$


This gives

$$
C(U, W)=\frac{\tilde{C}(u, W)}{\dot{p}(u)},
$$

where, for events lying outside the collapsing star, $\tilde{C}(u, W)$ is the metric coefficient of a static spacetime. For any massless quantum field, the renormalised stress-energy-momentum tensor corresponding to a quantum state that behaves like the Boulware vacuum asymptotically in the past has components [26]

$$
T_{U U} \propto C^{1 / 2} \partial_{U}^{2} C^{-1 / 2}, \quad T_{W W} \propto C^{1 / 2} \partial_{W}^{2} C^{-1 / 2}, \quad T_{U W} \propto C R,
$$

where $R$ is the curvature scalar, and the numerical coefficients depend on the specific type of field being considered.

The component with the most interesting structure is $T_{U U}$. Using equation (4.11) and the property $\partial_{U}=\dot{p}^{-1} \partial_{u}$ one finds

$$
C^{1 / 2} \partial_{U}^{2} C^{-1 / 2}=\frac{1}{\dot{p}^{2}}\left(\tilde{C}^{1 / 2} \partial_{u}^{2} \tilde{C}^{-1 / 2}-\dot{p}^{1 / 2} \partial_{u}^{2} \dot{p}^{-1 / 2}\right)
$$

The key point is that the first term within brackets on the right hand side of equation (4.13) is a static contribution due to the Boulware-like modes, while the second one arises because of the dynamics of collapse. These two terms, separately, would lead to an arbitrarily large $T_{U U}$ as the horizon is approached, because $\dot{p}$ tends to vanish in this limit. However, if (and only if) the horizon forms, then the leading contributions of $\tilde{C}^{1 / 2} \partial_{u}^{2} \tilde{C}^{-1 / 2}$ and $\dot{p}^{1 / 2} \partial_{u}^{2} \dot{p}^{-1 / 2}$ exactly cancel against each other.

For the computation, it is convenient to work in a chart that is regular at the horizon (if it forms), so that the regularity of the stress-energy-momentum tensor can be inferred just by the finiteness of its components. This is the case for the Painlevé-Gullstrand coordinates $(x, t)$, in terms of which the metric is $[27,28,29]$ :

$$
g=-c(x, t)^{2} \mathrm{~d} t^{2}+(\mathrm{d} x-v(x, t) \mathrm{d} t)^{2} .
$$

A rather technical computation gives [10]:

$$
\begin{aligned}
T_{t t} & =U_{t}^{2} T_{U U}+2 U_{t} W_{t} T_{U W}+W_{t}^{2} T_{W W} \\
& =(c+v)^{2} U_{x}^{2} T_{U U}-2\left(c^{2}-v^{2}\right) U_{x} W_{x} T_{U W}+(c-v)^{2} W_{x}^{2} T_{W W} \\
& =\dot{p}^{2} T_{U U}-2 \dot{p} T_{U W}+T_{W W} \\
T_{t x} & =U_{t} U_{x} T_{U U}+\left(U_{t} W_{x}+U_{x} W_{t}\right) T_{U W}+W_{t} W_{x} T_{W W} \\
& =-(c+v) U_{x}^{2} T_{U U}-2 v U_{x} W_{x} T_{U W}+(c-v) W_{x}^{2} T_{W W} \\
& =-\frac{\dot{p}^{2}}{c+v} T_{U U}+\frac{2 \dot{p} v}{c^{2}-v^{2}} T_{U W}+\frac{1}{c-v} T_{W W} \\
T_{x x} & =U_{x}^{2} T_{U U}+2 U_{x} W_{x} T_{U W}+W_{x}^{2} T_{W W} \\
& =\frac{\dot{p}^{2}}{(c+v)^{2}} T_{U U}-2 \frac{\dot{p}}{c^{2}-v^{2}} T_{U W}+\frac{1}{(c-v)^{2}} T_{W W} .
\end{aligned}
$$


Since at a horizon $c+v \rightarrow 0$ and $c-v \rightarrow 2 c$, the term that presents, potentially, the highest degree of divergence is the one proportional to $T_{U U}$ in $T_{x x}$. The other potentially dangerous coefficients $\dot{p}^{-2}$ and $\dot{p}^{-1}$ that appear in $T_{U U}$ and $T_{U W}$ are cancelled by corresponding factors in the expressions (4.17), (4.20), and (4.22).

In the rest of the calculations, we assume that $c(x)=1$ and place the horizon (when it exists) at $x=0$. Then, assuming that a horizon indeed forms, we can expand $v(x)$ as

$$
v(x)=-1+\kappa x+\mathscr{O}\left(x^{2}\right),
$$

where $\kappa$ can be identified with the surface gravity $[28,29]$. The static contribution in equation 4.13 is

$$
\tilde{C}^{1 / 2} \partial_{u}^{2} \tilde{C}^{-1 / 2}=\frac{\kappa^{2}}{4}+\mathscr{O}\left(x^{2}\right)
$$

and this, taken alone, would cause the $T_{t x}$ and $T_{x x}$ coefficients to diverge. However, under the hypothesis of horizon formation one also has [10]

$$
p(u)=U_{\mathrm{H}}-A_{1} \mathrm{e}^{-\kappa u}+\frac{A_{2}}{2} \mathrm{e}^{-2 \kappa u}+\frac{A_{3}}{3 !} \mathrm{e}^{-3 \kappa u}+\mathscr{O}\left(\mathrm{e}^{-4 \kappa u}\right),
$$

where $U_{\mathrm{H}}, A_{1}>0, A_{2}, A_{3}$ are constants. The dynamical term in equation (4.13) is then

$$
\dot{p}^{1 / 2} \partial_{u}^{2} \dot{p}^{-1 / 2}=\frac{\kappa^{2}}{4}+\left[-\frac{1}{2} \frac{A_{3}}{A_{1}}+\frac{3}{4}\left(\frac{A_{2}}{A_{1}}\right)^{2}\right] \kappa^{2} \mathrm{e}^{-2 \kappa u}+\mathscr{O}\left(\mathrm{e}^{-3 \kappa u}\right) .
$$

Replacing the expressions (4.24) and (4.26) into equation (4.13), one sees that the dominant terms $\kappa^{2} / 4$ cancel against each other, and one remains with a finite contribution that depends on the details of collapse:

$$
T_{U U}^{\text {assuming horizon formation }} \propto \frac{1}{\dot{p}^{2}}\left[-\frac{1}{2} \frac{A_{3}}{A_{1}}+\frac{3}{4}\left(\frac{A_{2}}{A_{1}}\right)^{2}\right] \kappa^{2} \mathrm{e}^{-2 \kappa u}+\ldots,
$$

so that

$$
T_{x x}^{\text {assuming horizon formation }} \propto\left[-\frac{1}{2} \frac{A_{3}}{A_{1}}+\frac{3}{4}\left(\frac{A_{2}}{A_{1}}\right)^{2}\right] \frac{1}{x^{2}} \mathrm{e}^{-2 \kappa u}+\ldots,
$$

with these expressions holding outside the surface of the collapsing star. Furthermore, it is easy to realise that this contribution is inversely proportional to the square of the speed at which the collapsing body crosses its gravitational radius. Hence, for a very slow collapse there is a realistic and concrete possibility that the (energy-condition-violating) renormalised stress-energy-momentum tensor, although finite, could lead to significant deviations from classical collapse when a trapping horizon is just about to form.

In order to reinforce this claim, let us consider a case in which the horizon never forms at any finite time, but is only approached asymptotically in the limit $t \rightarrow+\infty$. In particular, we shall be interested in an exponential approach [29], where the radius of the star depends on time as

$$
r(t)=2 M+B \mathrm{e}^{-\kappa_{\mathrm{D}} t},
$$


with $B$ and $\kappa_{\mathrm{D}}$ positive constants. After a brief calculation [10,29] this leads to

$$
p(u)=U_{\mathrm{H}}-A_{1} \mathrm{e}^{-\kappa_{\text {eff }} u},
$$

where

$$
\kappa_{\text {eff }}=\frac{\kappa \kappa_{\mathrm{D}}}{\kappa+\kappa_{\mathrm{D}}}<\kappa
$$

Here $\kappa_{\text {eff }}$ can be thought of as a "reduced surface gravity". Interestingly, although no true horizon ever forms, one still gets a Hawking-like flux of Planckian radiation at the temperature [29]

$$
T=\frac{\kappa_{\mathrm{eff}}}{2 \pi}=\frac{\kappa \kappa_{\mathrm{D}}}{2 \pi\left(\kappa+\kappa_{\mathrm{D}}\right)} .
$$

Of course, outside the star, the calculation of $\tilde{C}^{1 / 2} \partial_{u}^{2} \tilde{C}^{-1 / 2}$ again yields the same result as in equation (4.24). However, for the second contribution we now have

$$
\dot{p}^{1 / 2} \partial_{u}^{2} \dot{p}^{-1 / 2}=\frac{\kappa_{\text {eff }}^{2}}{4}+\mathscr{O}\left(\mathrm{e}^{-2 \kappa_{\mathrm{eff}} u}\right),
$$

so there is no longer a perfect cancellation between the dominant terms in the static and dynamical contributions. Indeed, at the leading order, the renormalised stress-energy-momentum tensor outside the star in the limit $x \rightarrow 0$ (that is, $r \rightarrow 2 M$, or $t \rightarrow+\infty$ ) behaves as

$$
T_{U U} \propto \frac{\kappa_{\mathrm{eff}}^{2}-\kappa^{2}}{4 \dot{p}^{2}}+\ldots
$$

so that

$$
T_{x x} \propto \frac{\kappa_{\text {eff }}^{2}-\kappa^{2}}{4 \kappa^{2} x^{2}}+\ldots
$$

Note that this result is not in contradiction with the Fulling-Sweeny-Wald theorem [17], because a strict divergence appears only for $t=+\infty$, i.e., at the boundary of spacetime. However, the renormalised stress-energy-momentum tensor gives an arbitrarily large (albeit finite) energy-conditionviolating contribution to the right hand side of the semiclassical Einstein equations as the horizon formation condition $2 M / r=1$ is approached.

\section{Implications and Discussion}

In the standard collapse, you can argue that the renormalised stress-energy-momentum tensor at horizon-crossing, as felt by infalling matter, is negligible provided:

1. the quantum state is of the Hadamard form (which we have by assertion);

2. matter is basically freely-falling;

3. the equivalence principle holds.

The first point tells you that the quantum vacuum has the same ultraviolet form as in Minkowski spacetime, the second point tells you that matter is approximately in a locally inertial frame, and the third point tells you that the local renormalised stress-energy-momentum tensor the matter then 


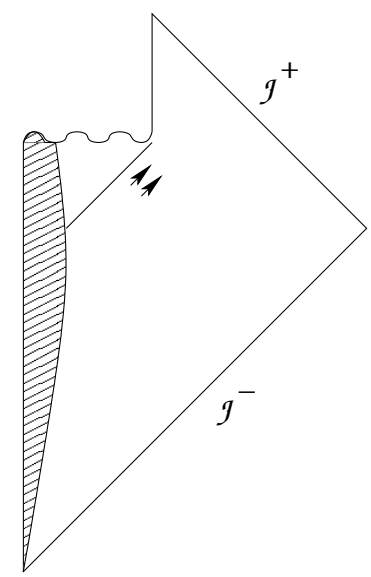

Figure 4: Standard Carter-Penrose diagram for an evaporating black hole. Don't bother asking what happens at the endpoint of the evaporation process — in the standard causal picture there is no definite answer.

"feels" must be approximately the same as in Minkowski spacetime - i.e., approximately zero (after renormalization).

In contrast, our result is saying that large deviations from this standard conclusion can arise if matter is not freely falling, but is significantly accelerated (as, by self-consistency, it must be to sustain itself against the gravitational attraction). So we are explicitly violating point 2, (while we explicitly keep point 1 , and implicitly keep point 3 ). So if the surface of the star deviates significantly from free-fall, then a large stress-energy-momentum builds up, which can force it further away from free-fall - either stopping or exponentially delaying the collapse. Precisely predicting what happens in a specific collapse scenario relies on extremely messy model-dependent physics.

Indeed our calculation seems to suggest that if, during the late stages of the collapse, matter is far from free-fall, then a growing RSET can lead to a late time collapse history very different from the classically expected one, possibly leading to a form of asymptotic collapse of the type suggested in (4.29). It might even be that this is the solution preferred by nature; this might be due to new particle physics effect coming into place in the late stages of most stellar collapses. In this case the conformal diagram describing the gravitational collapse scenario would not be the standard one of figure 4, but rather that reported in figure 5. This object would then be a "quasi-black hole" (not to be confused with the homonymous objects proposed by Lemos and Zaslavskii [30], which are static solutions), an object which would not only closely mimic the classical geometry of a black hole, but also, (if the collapse law is exponential at late times), mimic its quantum effects such as Hawking radiation.

To place our results in a broader perspective: Many physicists are now (for numerous independent reasons) arguing against the standard Carter-Penrose diagram, figure 4, for the formation and evaporation of a semi-classical black hole. Apart from the "physics challenged", (whom we shall quietly discount), there are hints from analogue spacetimes [29], from loop quantum gravity [31], 


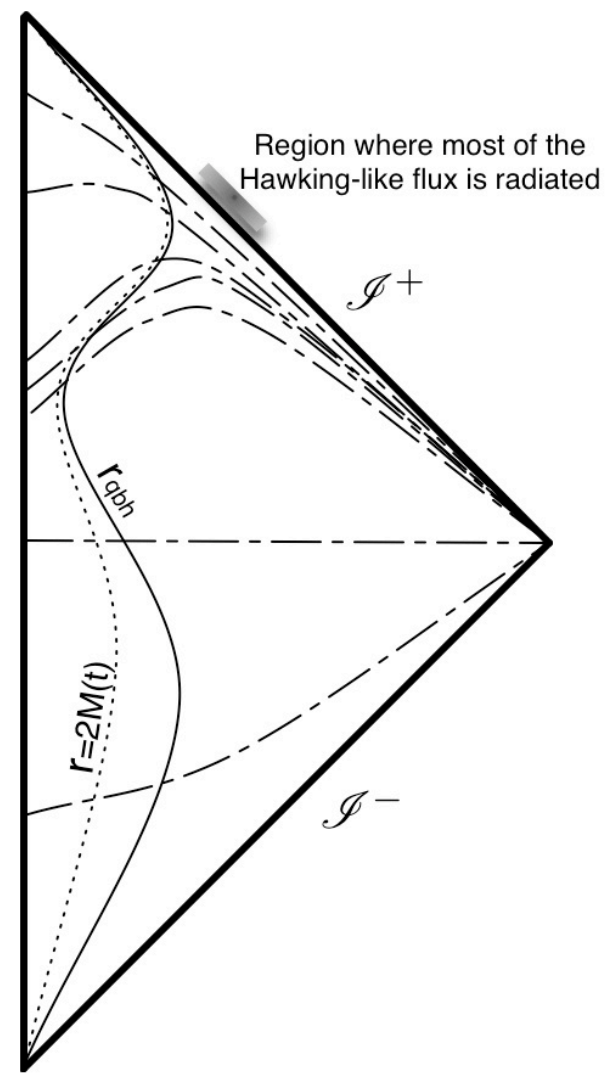

Figure 5: Conformal diagram of the spacetime for a quasi-black hole. The solid line represents the surface of the collapsing object; the dotted line is at $r=2 M(t)$, where $M(t)$ is the instantaneous mass of the object as measured from $\mathscr{I}^{+}$; dashed lines correspond to (Schwarzschild) $t=$ const hypersurfaces. The period of evaporation appears short because of a distortion induced by the representation, but actually corresponds to a very long lapse of time, as one can see from the fact that the lines at $t=$ const crowd around it. This diagram, while nonstandard, is nevertheless compatible with current astrophysical observations of gravitationally active collapse products.

from string-inspired models [32], from attempts at unitarity preservation in our own domain of outer communication [33], from one-loop curved-space quantum field theory [34], and from abstract studies of the nature of horizons [35], all hinting at a more subtle history for collapse and evolution. (Canonical versions of alternative causal structures are given by the Carter-Penrose diagrams of figures 6 and 7, and the double-null diagram of figure 8.) Unfortunately, when attempting to move beyond qualitative statements of this type, specific predictions are frustratingly model-dependent, but there is some "wriggle room" for interesting new physics.

On a cautionary note, we should point out that several authors have looked at the question of what observational signals for black hole mimics might look like [36, 37]. Critically, once you add rotation, the ergosurface is probably more important than the "would-be horizon". There is the very real risk of significant ergoregion instabilities [38].

In summary, what our calculation suggests is that it might be possible to have a black hole 


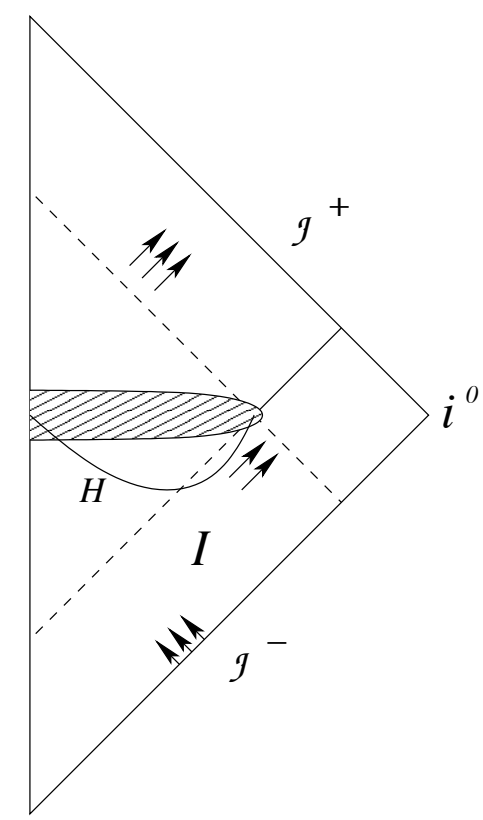

Figure 6: Ashtekar-Bojowald version of the Carter-Penrose diagram for an evaporating black hole. The shaded region represents a region of Planck-scale curvature, and possibly large metric fluctuations.

without having a black hole - a configuration that is a black hole for (almost) all practical purposes, but might be missing the one key ingredient of having a horizon. Deep issues of principle remain, and it will be very interesting to see how the whole area of black hole mimics develops over the next few years.

\section{References}

[1] B. Freedman and L. D. McLerran, Quark star phenomenology, Phys. Rev. D 17, 1109-1122 (1978). J. J. Drake et al., Is RX J1856.5-3754 a quark star?, Astrophys. J. 572, 996-1101 (2002) [arXiv:astro-ph/0204159].

[2] S. R. Coleman, Q-balls, Nucl. Phys. B 262, 263-283 (1985). Erratum: ibid. 269, 744 (1986).

[3] C. Alcock, E. Farhi and A. Olinto, Strange stars, Astrophys. J. 310, 261-272 (1986).

P. Haensel, J. L. Zdunik and R. Schaeffer, Strange quark stars, Astron. Astrophys. 160, 121-128 (1986).

X. D. Li, I. Bombaci, M. Dey, J. Dey and E. P. J. van den Heuvel, Is SAX J1808.4-3658 a strange star?, Phys. Rev. Lett. 83, 3776-3779 (1999) [arXiv:hep-ph/9905356].

[4] M. Colpi, S. L. Shapiro and I. Wasserman, Boson stars: Gravitational equilibria of self-interacting scalar fields, Phys. Rev. Lett. 57, 2485-2488 (1986).

P. Jetzer, Boson stars, Phys. Rept. 220, 163-227 (1992). 

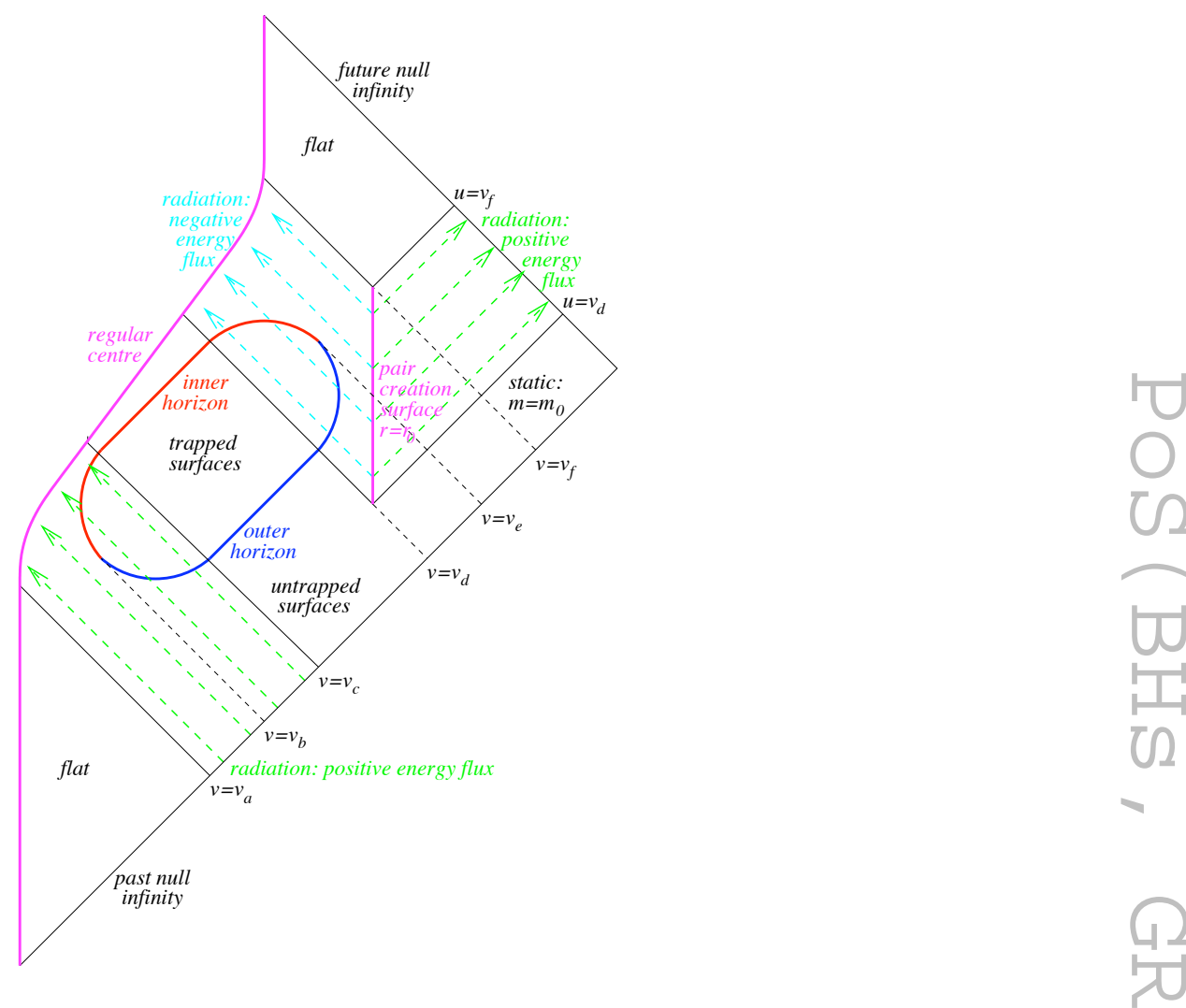

Figure 7: Hayward version of the Carter-Penrose diagram for an evaporating black hole.
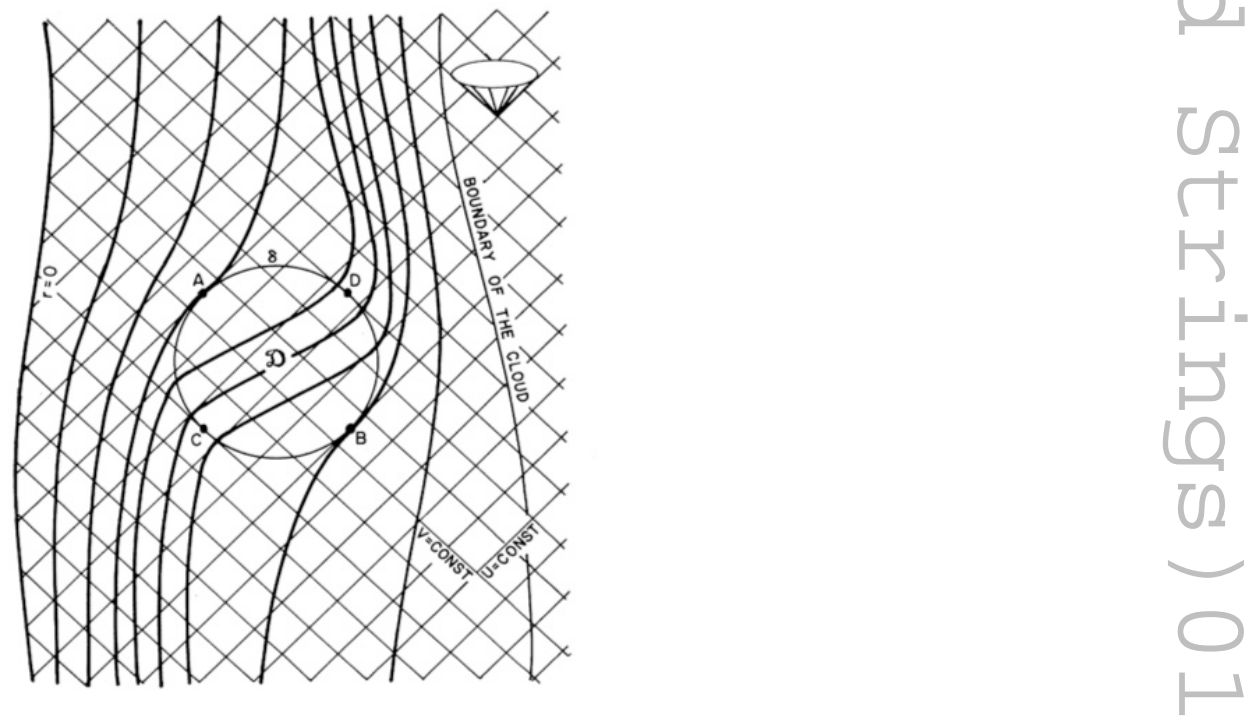

Figure 8: Bergmann-Roman double-null causal diagram for a regular collapsing star. 
[5] P. O. Mazur and E. Mottola, Gravitational condensate stars: An alternative to black holes, arXiv:gr-qc/0109035.

P. O. Mazur and E. Mottola, Dark energy and condensate stars: Casimir energy in the large, in Quantum Field Theory Under the Influence of External Conditions, edited by K. A. Milton (Rinton Press, Princeton, 2004), pp. 350-357 [arXiv:gr-qc/0405111].

P. O. Mazur and E. Mottola, Gravitational vacuum condensate stars, Proc. Nat. Acad. Sci. 101, 9545-9550 (2004) [arXiv:gr-qc/0407075].

P. O. Mazur and E. Mottola, Dark energy and condensate stars: A quantum alternative to classical black holes, in Proceedings of the Tenth Marcel Grossmann Meeting on General Relativity, Part B, edited by S. Perez Bergliaffa and M. Novello (World Scientific, Singapore, 2006), pp. 1041-1047.

[6] G. Chapline, E. Hohlfeld, R. B. Laughlin and D. I. Santiago, Quantum phase transitions and the breakdown of classical general relativity, Int. J. Mod. Phys. A 18, 3587-3590 (2003)

[arXiv:gr-qc/0012094].

R. B. Laughlin, Emergent relativity, Int. J. Mod. Phys. A 18, 831-854 (2003) [arXiv:gr-qc/0302028].

[7] O. Lunin and S. D. Mathur, Statistical interpretation of Bekenstein entropy for systems with a stretched horizon, Phys. Rev. Lett. 88, 211303 (2002) [arXiv:hep-th/0202072].

S. D. Mathur, The fuzzball proposal for black holes: An elementary review, Fortsch. Phys. 53, 793-827 (2005) [arXiv:hep-th/0502050].

S. Giusto and S. D. Mathur, Fuzzball geometries and higher derivative corrections for extremal holes, Nucl. Phys. B 738, 48-75 (2006) [arXiv:hep-th/0412133].

B. D. Chowdhury and S. D. Mathur, Radiation from the non-extremal fuzzball, Class. Quantum Grav. 25, 135005 (2008) [arXiv:0711.4817 [hep-th]].

S. D. Mathur, Tunneling into fuzzball states, arXiv:0805.3716 [hep-th].

B. D. Chowdhury and S. D. Mathur, Pair creation in non-extremal fuzzball geometries, Class. Quantum Grav. 25, 225021 (2008) [arXiv:0806.2309 [hep-th]].

S. D. Mathur, Fuzzballs and the information paradox: a summary and conjectures, arXiv:0810.4525 [hep-th].

[8] D. Amati, How strings solve the apparent contradiction between black holes and quantum coherence, J. Phys. G 29, 31-34 (2003) [arXiv:gr-qc/0103073].

D. Amati, The information paradox, in String Theory and Fundamental Interactions, edited by M. Gasperini and J. Maharana, Lecture Notes in Physics 737, 609 (Berlin, Springer, 2008) [arXiv:hep-th/0612061].

[9] K. Skenderis and M. Taylor, The fuzzball proposal for black holes, Phys. Rept. 467 (2008) 117 [arXiv:0804.0552 [hep-th]].

[10] C. Barceló, S. Liberati, S. Sonego and M. Visser, Fate of gravitational collapse in semiclassical gravity, Phys. Rev. D 77, 044032 (2008) [arXiv:0712.1130 [gr-qc]].

[11] C. Barceló, S. Liberati, S. Sonego and M. Visser, Revisiting the semiclassical gravity scenario for gravitational collapse, Proceedings of ERE2008, the Spanish Relativity Meeting of 2008 held in Salamanca.

[12] D. G. Boulware, Hawking radiation and thin shells, Phys. Rev. D 13, 2169-2187 (1976).

[13] T. Vachaspati, D. Stojkovic and L. M. Krauss, Observation of incipient black holes and the information loss problem, Phys. Rev. D 76, 024005 (2007) [arXiv:gr-qc/0609024].

T. Vachaspati and D. Stojkovic, Quantum radiation from quantum gravitational collapse, Phys. Lett. B 663, 107-110 (2008) [arXiv:gr-qc/0701096]. 
T. Vachaspati, Schrodinger picture of quantum gravitational collapse, arXiv:0711.0006 [gr-qc].

[14] P. Hájíček, Classical horizons and quantum corrections, Phys. Lett. B 182, 309-311 (1986).

P. Hájíček, On the origin of Hawking radiation, Phys. Rev. D 36, 1065-1079 (1987).

[15] P. Hájíček, What simplified models say about unitarity and gravitational collapse, Nucl. Phys. Proc. Suppl. 88, 114-123 (2000) [arXiv:gr-qc/9912064].

P. Hájíček and C. Kiefer, Singularity avoidance by collapsing shells in quantum gravity, Int. J. Mod. Phys. D 10, 775-780 (2001) [arXiv:gr-qc/0107102].

[16] B. K. Harrison, K. S. Thorne, M. Wakano, and J. A. Wheeler, Gravitation Theory and Gravitational Collapse (University of Chicago Press, Chicago, 1965).

[17] S. A. Fulling, M. Sweeny and R. M. Wald, Singularity structure of the two-point function in quantum field theory in curved space-time, Commun. Math. Phys. 63, 257-264 (1978).

[18] S. W. Hawking and G. F. R. Ellis, The Large Scale Structure of Space-Time (Cambridge University Press, Cambridge, 1973).

C. W. Misner, K. S. Thorne, and J. A. Wheeler, Gravitation (Freeman, San Francisco, 1973).

R. M. Wald, General Relativity (University of Chicago Press, Chicago, 1984).

J. B. Hartle, Gravity: An Introduction to Einstein's General Relativity (Benjamin Cummings, 2003).

S. M. Carroll, Spacetime and Geometry: An Introduction to General Relativity (Benjamin Cummings, 2003).

[19] M. Visser, Lorentzian Wormholes: From Einstein to Hawking (AIP Press [now Springer-Verlag], New York, 1995).

[20] A. Fabbri and J. Navarros-Salas, Modelling Black Hole Evaporation (Imperial College Press, London, 2005).

[21] A. Ashtekar and B. Krishnan, Dynamical horizons: Energy, angular momentum, fluxes and balance laws, Phys. Rev. Lett. 89, 261101 (2002) [arXiv:gr-qc/0207080].

A. Ashtekar and B. Krishnan, Dynamical horizons and their properties, Phys. Rev. D 68, 104030 (2003) [arXiv:gr-qc/0308033].

A. Ashtekar and B. Krishnan, Isolated and dynamical horizons and their applications, Living Rev.

Rel. 7, 10 (2004); URL (cited on 29 January 2009):

http: / / www. livingreviews . org/lrr-2004-10 [arXiv:gr-qc/0407042].

A. Ashtekar and G. J. Galloway, Some uniqueness results for dynamical horizons, Adv. Theor. Math. Phys. 9, 1-30 (2005) [arXiv:gr-qc/0503109].

A. Ashtekar, Black hole dynamics in general relativity, Pramana 69, 77-92 (2007).

[22] S. A. Hayward, Marginal surfaces and apparent horizons, arXiv:gr-qc/9303006.

S. A. Hayward, General laws of black hole dynamics, Phys. Rev. D 49, 6467-6474 (1994).

S. A. Hayward, Unified first law of black-hole dynamics and relativistic thermodynamics, Class. Quantum Grav. 15, 3147-3162 (1998) [arXiv:gr-qc/9710089].

S. A. Hayward, Black holes: New horizons, in Proceedings of the Ninth Marcel Grossmann Meeting on General Relativity, Part A, edited by V. G. Gurzadyan, R. T. Jantzen and R. Ruffini (World Scientific, Singapore, 2002), pp. 568-580 [arXiv:gr-qc/0008071].

S. A. Hayward, Energy conservation for dynamical black holes, Phys. Rev. Lett. 93, 251101 (2004) [arXiv:gr-qc/0404077].

S. A. Hayward, Energy and entropy conservation for dynamical black holes, Phys. Rev. D 70, 104027 (2004) [arXiv:gr-qc/0408008]. 
S. A. Hayward, Gravitational radiation from dynamical black holes, Class. Quantum Grav. 23, L15-L21 (2006) [arXiv:gr-qc/0505080].

S. A. Hayward, Angular momentum conservation for dynamical black holes, Phys. Rev. D 74, 104013 (2006) [arXiv:gr-qc/0609008].

S. A. Hayward, Dynamics of black holes, arXiv:0810.0923 [gr-qc].

[23] J. Massó, J. Libson, E. Seidel and W. M. Suen, A 3-D apparent horizon finder, in The Seventh Marcel Grossmann Meeting, edited by R. T. Jantzen and G. Mac Keiser (World Scientific, Singapore, 1997), pp. 631-633 [arXiv:gr-qc/9412058].

T. W. Baumgarte, G. B. Cook, M. A. Scheel, S. L. Shapiro and S. A. Teukolsky, Implementing an apparent-horizon finder in three dimensions, Phys. Rev. D 54, 4849-4857 (1996) [arXiv:gr-qc/9606010].

M. Alcubierre, S. Brandt, B. Brügmann, C. Gundlach, J. Massó, E. Seidel and P. Walker, Test-beds and applications for apparent horizon finders in numerical relativity, Class. Quantum Grav. 17, 2159-2190 (2000) [arXiv:gr-qc/9809004].

[24] A. B. Nielsen and M. Visser, Production and decay of evolving horizons, Class. Quantum Grav. 23, 4637-4658 (2006) [arXiv:gr-qc/0510083].

A. B. Nielsen, Black holes as local horizons, arXiv:0711.0313 [gr-qc].

A. B. Nielsen and J. H. Yoon, Dynamical surface gravity, Class. Quantum Grav. 25, 085010 (2008) [arXiv:0711.1445 [gr-qc]].

A. B. Nielsen, Black holes without event horizons, arXiv:0802.3422 [gr-qc].

A. B. Nielsen and D. H. Yeom, Spherically symmetric trapping horizons, the Misner-Sharp mass and black hole evaporation, arXiv:0804.4435 [gr-qc].

A. B. Nielsen, Black holes without boundaries, arXiv:0809.1711 [gr-qc].

A. B. Nielsen, Black holes and black hole thermodynamics without event horizons, arXiv:0809.3850 [hep-th].

[25] J. Thornburg, Event and apparent horizon finders for $3+1$ numerical relativity, Living Rev. Rel. 10, 3 (2007); URL (cited on 29 January 2009): http: / / www . livingreviews . org/ Irr-2007-3 [arXiv:gr-qc/0512169].

[26] P. C. W. Davies, S. A. Fulling and W. G. Unruh, Energy-momentum tensor near an evaporating black hole, Phys. Rev. D 13, 2720-2723 (1976).

S. M. Christensen and S. A. Fulling, Trace anomalies and the Hawking effect, ibid. 15, 2088-2104 (1977).

P. C. W. Davies and S. A. Fulling, Quantum vacuum energy in two dimensional space-times, Proc. R. Soc. Lond. A 354, 59-77 (1977).

[27] P. Painlevé, La mécanique classique et la theorie de la relativité, C. R. Acad. Sci. (Paris) 173, 677-680 (1921).

A. Gullstrand, Allgemeine Lösung des statischen Einkörperproblems in der Einsteinschen Gravitationstheorie, Ark. Mat. Astron. Fys. 16, 1-15 (1922).

[28] C. Barcelò, S. Liberati, S. Sonego and M. Visser, Causal structure of acoustic spacetimes, New J. Phys. 6, 186 (2004) [arXiv:gr-qc/0408022].

C. Barceló, S. Liberati and M. Visser, Analogue gravity, Living Rev. Relativity 8, 12 (2005); URL (cited on 29 January 2009): http: / / www . livingreviews . org/lrr-2005-12 [arXiv:gr-qc/0505065]. 
[29] C. Barceló, S. Liberati, S. Sonego and M. Visser, Quasi-particle creation by analogue black holes, Class. Quantum Grav. 23, 5341-5366 (2006) [arXiv:gr-qc/0604058].

C. Barceló, S. Liberati, S. Sonego and M. Visser, Hawking-like radiation does not require a trapped region, Phys. Rev. Lett. 97, 171301 (2006) [arXiv:gr-qc/0607008].

[30] J. P. S. Lemos and O. B. Zaslavskii, The angular momentum and mass formulas for rotating stationary quasi-black holes, arXiv:0901.3860 [gr-qc].

J. P. S. Lemos and O. B. Zaslavskii, The mass formula for quasi-black holes, Phys. Rev. D 78 (2008) 124013 [arXiv:0811.2778 [gr-qc]].

J. P. S. Lemos and O. B. Zaslavskii, Quasi black holes: definition and general properties, Phys. Rev. D 76 (2007) 084030 [arXiv:0707.1094 [gr-qc]].

[31] A. Ashtekar and M. Bojowald, Black hole evaporation: a paradigm, Class. Quantum Grav. 22, 3349-3362 (2005) [arXiv:gr-qc/0504029].

[32] D. Amati and J. G. Russo, Fundamental strings as black bodies, Phys. Lett. B 454, 207-212 (1999) [arXiv:hep-th/9901092].

D. Amati, M. Ciafaloni and G. Veneziano, Towards an S-matrix description of gravitational collapse, JHEP 0802, 049 (2008) [arXiv:0712.1209 [hep-th]].

[33] S. W. Hawking, Information loss in black holes, Phys. Rev. D 72, 084013 (2005) [arXiv:hep-th/0507171].

[34] E. Mottola and R. Vaulin, Macroscopic effects of the quantum trace anomaly, Phys. Rev. D 74, 064004 (2006) [arXiv:gr-qc/0604051].

P. R. Anderson, E. Mottola and R. Vaulin, Stress tensor from the trace anomaly in Reissner-Nordstrom spacetimes, Phys. Rev. D 76, 124028 (2007) [arXiv:0707.3751 [gr-qc]].

P. R. Anderson, M. Binkley, H. Calderon, W. A. Hiscock, E. Mottola and R. Vaulin, Effects of quantized fields on the spacetime geometries of static spherically symmetric black holes, in Proceedings of the Eleventh Marcel Grossmann Meeting on General Relativity, edited by H. Kleinert and R. T. Jantzen (World Scientific, Singapore, 2008), pp. 1497-1499 [arXiv:0709.4457 [gr-qc]].

[35] T. A. Roman and P. G. Bergmann, Stellar collapse without singularities?, Phys. Rev. D 28, 1265-1277 (1983).

[36] C. B. M. Chirenti and L. Rezzolla, How to tell a gravastar from a black hole, Class. Quantum Grav. 24, 4191-4206 (2007) [arXiv:0706.1513 [gr-qc]].

[37] A. E. Broderick and R. Narayan, Where are all the gravastars? Limits upon the gravastar model from accreting black holes, Class. Quantum Grav. 24, 659-666 (2007) [arXiv:gr-qc/0701154].

[38] V. Cardoso, P. Pani, M. Cadoni and M. Cavaglià, Ergoregion instability of ultracompact astrophysical objects, Phys. Rev. D 77, 124044 (2008) [arXiv:0709.0532 [gr-qc]].

V. Cardoso, P. Pani, M. Cadoni and M. Cavaglià, Instability of hyper-compact Kerr-like objects, Class. Quantum Grav. 25, 195010 (2008) [arXiv:0808.1615 [gr-qc]].

C. B. M. Chirenti and L. Rezzolla, On the ergoregion instability in rotating gravastars, Phys. Rev. D 78, 084011 (2008) [arXiv:0808.4080 [gr-qc]].

P. Pani, V. Cardoso, M. Cadoni and M. Cavaglià, Ergoregion instability of black hole mimickers, arXiv:0901.0850 [gr-qc]. 